\title{
Effects of correlated hopping on electronic ferroelectricity in the extended Falicov-Kimball model in two dimensions
}

\author{
P. Farkašovskŷ:, J. Jurečková \\ Institute of Experimental Physics, Slovak Academy of Sciences, Watsonova 47, 04001 Košice, Slovakia
}

Received April 29, 2015

\begin{abstract}
We use the Hartree-Fock (HF) approximation with the charge-density-wave (CDW) instability to study the effect of correlated hopping on the stability of electronic ferroelectricity in the extended Falicov-Kimball model (FKM) in two dimensions. It is shown that the effects of correlated hopping term are very strong, especially for negative values of the $f$-level energy $E_{f}$, where they lead to: (i) stabilization of the ferroelectric ground state with a spontaneous hybridization $P_{d f}=\left\langle d^{+} f\right\rangle$ for positive values of correlated hopping parameter $t_{d^{\prime}}^{\prime}$ (ii) stabilization of the antiferroelectric phase for $t_{d}^{\prime}<t_{d \text {,crit. }}^{\prime}<0$ and (iii) suppression of the ferroelectric ground state for $t_{d, \text { crit. }}^{\prime} \leqslant t_{d}^{\prime}<0$, The effects of correlated hopping on valence transitions are also discussed.
\end{abstract}

Key words: electronic ferroelectricity, charge density waves, valence transitions

PACS: $71.27 .+a, 71.28 .+d, 71.30 .+h$

\section{Introduction}

In the recent years the Falicov-Kimball model (FKM) [1] has been extensively studied in connection with the exciting idea of electronic ferroelectricity [2-11] that is also directly related to the problem of an excitonic insulator [12-18]. It is generally supposed that ferroelectricity in mixed-valent compounds is of purely electronic origin, i.e., it results from an electronic phase transition, in contrast to the conventional displacive ferroelectricity due to a lattice distortion. Since the FKM is probably the simplest model of electronic phase transitions in rare-earth and transition-metal compounds it was natural to test the idea of electronic ferroelectricity just on this model.

The FKM is based on the coexistence of two different types of electronic states in a given material: localized, highly correlated ionic-like states and extended, uncorrelated, Bloch-like states. It is accepted that insulator-metal transitions result from a change in the occupation numbers of these electronic states, which remain themselves basically unchanged by their character. Taking into account only the intraatomic Coulomb interaction between the two types of states, the Hamiltonian of the spinless FKM can be written as the sum of three terms:

$$
H=\sum_{i j} t_{i j} d_{i}^{+} d_{j}+U \sum_{i} f_{i}^{+} f_{i} d_{i}^{+} d_{i}+E_{f} \sum_{i} f_{i}^{+} f_{i}
$$

where $f_{i}^{+}, f_{i}$ are the creation and annihilation operators for an electron in the localized state at lattice site $i$ with binding energy $E_{f}$, and $d_{i}^{+}, d_{i}$ are the creation and annihilation operators of the itinerant spinless electrons in the $d$-band Wannier state at site $i$.

\footnotetext{
*E-mail: farky@saske.sk
} 
The first term of (1) is the kinetic energy corresponding to quantum-mechanical hopping of the itinerant $d$ electrons between sites $i$ and $j$. These intersite hopping transitions are described by the matrix elements $t_{i j}$, which are $-t_{d}$ if $i$ and $j$ are the nearest neighbors and zero otherwise (in what follows all parameters are measured in units of $t_{d}$ ). The second term represents the on-site Coulomb interaction between the $d$-band electrons with density $n_{d}=\frac{1}{L} \sum_{i} d_{i}^{+} d_{i}$ and the localized $f$ electrons with density $n_{f}=\frac{1}{L} \sum_{i} f_{i}^{+} f_{i}$, where $L$ is the number of lattice sites. The third term stands for the localized $f$ electrons whose sharp energy level is $E_{f}$.

The first attempt to describe the electronic ferroelectricity within the FKM was made by Portengen et al. [2, 3]. They studied the FKM with a $k$-dependent hybridization in Hartree-Fock (HF) approximation and found, in particular, that the Coulomb interaction $U$ between the itinerant $d$-electrons and the localized $f$-electrons gives rise to a non-vanishing excitonic $\left\langle d^{+} f\right\rangle$-expectation value even in the limit of vanishing hybridization $V \rightarrow 0$. As an applied (optical) electrical field provides for excitations between $d$ - and $f$-states and thus for an excitonic expectation value $P_{d f}=\left\langle d_{i}^{+} f_{i}\right\rangle$, the finding of a spontaneous $P_{d f}$ (without hybridization or electric field) has been interpreted as the evidence for electronic ferroelectricity. However, analytical calculations within well controlled approximation (for $U$ small) performed by Czycholl [4] in infinite dimensions do not confirm the existence of electronic ferroelectricity. The same conclusion has been also obtained independently [5, 6] by small-cluster exact-diagonalization and density-matrix renormalization group calculations in the one dimension for both small $(U<1)$ and intermediate interactions $(U \sim 5)$.

Hybridization between the itinerant $d$ and localized $f$ states, however, is not the only way to develop $d-f$ coherence. Recent theoretical works by Batista et al. [8, 9] showed that the ground state with a spontaneous electric polarization can also be induced by the nearest-neighbor $f$-electron hopping $\left(-t_{f} \sum_{\langle i, j\rangle} f_{i}^{+} f_{j}\right)$ for dimensions $D>1$. Based on these results the authors postulated the following conditions that favor the formation of the electronically driven ferroelectric state: (i) the system should be in a mixed-valence regime, (ii) two bands involved should be of different parity, (iii) a local Coulomb repulsion $U$ between the $f$ and $d$ orbitals is required.

Later on this model was extensively used to describe different phases in the ground state and particularly the properties of the excitonic phase [12-19]. It was found that the ground state phase diagram exhibits a very simple structure consisting of only four phases, and namely, the full $d$ and $f$ band insulator (BI), the excitonic insulator (EI), the charge-density-wave (CDW) and the staggered orbital order (SOO). The EI is characterized by a nonvanishing $\left\langle d^{+} f\right\rangle$ average. The CDW is described by a periodic modulation in the total electron density of both $f$ and $d$ electrons, and the SOO is characterized by a periodic modulation in the difference between the $f$ and $d$ electron densities.

In our recent paper [10], the HF approach with the CDW instability was used to study the ground-state properties of the extended FKM with $f-f$ hopping in two and three dimensions. It was found that the HF solutions with the CDW instability perfectly reproduce the two-dimensional intermediate coupling phase diagram of the model calculated by constrained path Monte Carlo (CPMC) method [9], including phase boundaries of ferroelectric state. On the other hand, it should be noted that the model discussed above neglects all nonlocal interaction terms, and thus it is questionable whether the above mentioned results also persist in more realistic situations when nonlocal interactions are turned on. An important nonlocal interaction term obviously absent in the conventional FKM is the term of correlated hopping,

$$
H=-t_{d}^{\prime} \sum_{\langle i j\rangle} d_{i}^{+} d_{j}\left(f_{i}^{+} f_{i}+f_{j}^{+} f_{j}\right)
$$

in which the $d$-electron hopping amplitudes between the neighboring lattice sites $i$ and $j$ explicitly depend on the occupancy $\left(f_{i}^{+} f_{i}\right)$ of the $f$-electron orbitals. The importance of the correlated hopping term has been already mentioned by Hubbard [20]. Later Hirsch [21] pointed out that this term may be relevant in explaning the superconducting properties of strongly correlated electrons. Here, we examine the effects of this term on the stability of the EI phase in the two-dimensional extended FKM. With the exception of small deviations, the method used bellow is the same as the one described in our previous paper [10] and, therefore, we summarize here only its main steps. 


\section{The method}

The Hamiltonian of the extended FKM with correlated hopping has the form

$$
H=-t_{d} \sum_{\langle i j\rangle} d_{i}^{+} d_{j}-t_{f} \sum_{\langle i j\rangle} f_{i}^{+} f_{j}+U \sum_{i} f_{i}^{+} f_{i} d_{i}^{+} d_{i}+E_{f} \sum_{i} f_{i}^{+} f_{i}-t_{d}^{\prime} \sum_{\langle i j\rangle} d_{i}^{+} d_{j}\left(f_{i}^{+} f_{i}+f_{j}^{+} f_{j}\right) .
$$

In accordance with our previous paper [10], we go here beyond the usual HF approach [22] in which only homogeneous solutions are postulated and also consider inhomogeneous solutions modelled by a periodic modulation of the order parameters:

$$
\left\langle n_{i}^{f}\right\rangle=n_{f}+\delta_{f} \cos \left(\mathbf{Q} \cdot \mathbf{r}_{i}\right), \quad\left\langle n_{i}^{d}\right\rangle=n_{d}+\delta_{d} \cos \left(\mathbf{Q} \cdot \mathbf{r}_{i}\right), \quad\left\langle f_{i}^{+} d_{i}\right\rangle=\Delta+\Delta_{P} \cos \left(\mathbf{Q} \cdot \mathbf{r}_{i}\right) .
$$

Here, $\delta_{d}$ and $\delta_{f}$ are the order parameters of the CDW state for the $d$-and $f$-electrons, $\Delta$ is the excitonic average and $\mathbf{Q}=(\pi, \pi)$ is the nesting vector for $D=2$.

Using these expressions, the HF Hamiltonian of the extended FKM with correlated hopping can be written as

$$
\begin{aligned}
\mathscr{H}= & \left(-t_{d}-2 t_{d}^{\prime} n_{f}\right) \sum_{\langle i, j\rangle} d_{i}^{+} d_{j}-t_{f} \sum_{\langle i, j\rangle} f_{i}^{+} f_{j}+E_{f} \sum_{i} n_{i}^{f}+U \sum_{i}\left[n_{f}+\delta_{f} \cos \left(\mathbf{Q} \cdot \mathbf{r}_{i}\right)\right] n_{i}^{d} \\
& +U \sum_{i}\left[n_{d}+\delta_{d} \cos \left(\mathbf{Q} \cdot \mathbf{r}_{i}\right)\right] n_{i}^{f}-U \sum_{i}\left[\Delta+\Delta_{P} \cos \left(\mathbf{Q} \cdot \mathbf{r}_{i}\right)\right] d_{i}^{+} f_{i}+\text { h.c. },
\end{aligned}
$$

where the simple decoupling of the form

$$
d_{i}^{+} d_{j}\left(f_{i}^{+} f_{i}+f_{j}^{+} f_{j}\right) \rightarrow d_{i}^{+} d_{j}\left(\left\langle f_{i}^{+} f_{i}\right\rangle+\left\langle f_{j}^{+} f_{j}\right\rangle\right)=2 n_{f} d_{i}^{+} d_{j}
$$

has been used for the correlated hopping term. Thus, one can see that within this decoupling scheme, the effect of the correlated hopping is fully transformed to the renormalization of the static single electron hopping amplitude according to $t_{d} \rightarrow t_{d}^{*}=t_{d}+2 t_{d}^{\prime} n_{f}$. Next, we shall show that this relatively small change has dramatical effects on the existence of the EI phase in the extended FKM.

This Hamiltonian can be diagonalized by the following canonical transformation [10, 23]

$$
\gamma_{k}^{m}=u_{k}^{m} d_{k}+v_{k}^{m} d_{k+Q}+a_{k}^{m} f_{k}+b_{k}^{m} f_{k+Q}, \quad m=1,2,3,4,
$$

where $\Psi_{k}^{m}=\left(a_{k}^{m}, b_{k}^{m}, u_{k}^{m}, v_{k}^{m}\right)^{T}$ are solutions of the associated Bogoliubov-de Gennes (BdG) eigenequations:

$$
H_{k} \Psi_{k}^{m}=E_{k}^{m} \Psi_{k}^{m}
$$

with

$$
H_{k}=\left(\begin{array}{cccc}
\epsilon_{k}^{d}+U n_{f} & U \delta_{f} & -U \Delta & -U \Delta_{P} \\
U \delta_{f} & \epsilon_{k+Q}^{d}+U n_{f} & -U \Delta_{P} & -U \Delta \\
-U \Delta & -U \Delta_{P} & \epsilon_{k}^{f}+U n_{d}+E_{f} & U \delta_{d} \\
-U \Delta_{P} & -U \Delta & U \delta_{d} & \epsilon_{k+Q}^{f}+U n_{d}+E_{f}
\end{array}\right)
$$

and the corresponding energy dispersions $\epsilon_{k}^{d}$ and $\epsilon_{k}^{f}$ for $d$ and $f$ electrons and the HF parameters $n_{d}, \delta_{d}$, $n_{f}, \delta_{f}, \Delta, \Delta_{P}$ are given by:

$$
\begin{aligned}
\epsilon_{k}^{f}=-2 t_{f}\left[\cos \left(k_{x}\right)+\cos \left(k_{y}\right)\right], & \epsilon_{k}^{d}=-2 t_{d}^{*}\left[\cos \left(k_{x}\right)+\cos \left(k_{y}\right)\right], \\
n_{d}=\frac{1}{N} \sum_{k}^{\prime} \sum_{m}\left\{u_{k}^{m} u_{k}^{m}+v_{k}^{m} v_{k}^{m}\right\} f\left(E_{k}^{m}\right), & \delta_{d}=\frac{1}{N} \sum_{k}^{\prime} \sum_{m}\left\{v_{k}^{m} u_{k}^{m}+u_{k}^{m} v_{k}^{m}\right\} f\left(E_{k}^{m}\right), \\
n_{f}=\frac{1}{N} \sum_{k}^{\prime} \sum_{m}\left\{a_{k}^{m} a_{k}^{m}+b_{k}^{m} b_{k}^{m}\right\} f\left(E_{k}^{m}\right), & \delta_{f}=\frac{1}{N} \sum_{k}^{\prime} \sum_{m}\left\{b_{k}^{m} a_{k}^{m}+a_{k}^{m} b_{k}^{m}\right\} f\left(E_{k}^{m}\right), \\
\Delta=\frac{1}{N} \sum_{k}^{\prime} \sum_{m}\left\{a_{k}^{m} u_{k}^{m}+b_{k}^{m} v_{k}^{m}\right\} f\left(E_{k}^{m}\right), & \Delta_{P}=\frac{1}{N} \sum_{k}^{\prime} \sum_{m}\left\{b_{k}^{m} u_{k}^{m}+a_{k}^{m} v_{k}^{m}\right\} f\left(E_{k}^{m}\right) .
\end{aligned}
$$

Here, the prime denotes summation over half the Brillouin zone and $f(E)=1 /\{1+\exp [\beta(E-\mu)]\}$ is the Fermi distribution function. 


\section{Results and discussion}

To examine the effects of correlated hopping on the ground-state phase diagram of the extended FKM (in the $E_{f}-t_{f}$ plane), and particularly on the stability of the electronic ferroelectricity, we use the zero temperature variant of the method described above. The HF equations are solved self-consistently for each pair of $\left(E_{f}, t_{f}\right)$ values for several selected values of $t_{d}^{\prime}$. We use an exact diagonalization method to solve the Bogoliubov-de Gennes equation. We start with an initial set of the order parameters. By solving equation (8), the new order parameters are computed via equations (11) to (13) and are substituted back into equation (8). The iteration is repeated until a desired accuracy is achieved. It should be noted that the stability of different HF solutions has been also checked numerically by calculating the total energy and it was found that all phases presented in the ground-state phase diagram represent the most stable HF solutions.

First, we have examined the model in the intermediate coupling regime $U=2$, since for this case, there exists a comprehensive phase diagram of the extended FKM model ( $t_{f} \neq 0, t_{d}^{\prime}=0$ ) obtained by a CPMC technique [9] as well as its HF version [10] and both accord very nicely (both qualitatively and quantitatively). In figure 1 we have displayed typical examples of our HF solutions obtained for $\Delta, \Delta_{P}$ and $n_{f}$ in the limit of small $t_{f}$ values $\left(t_{f}=-0.1\right)$ and $t_{d}^{\prime}<0$. One can see that going with $t_{d}^{\prime}$ from 0 to $t_{d \text {,crit. }}^{\prime}=-0.5$, the effects of correlated hopping on the stability of excitonic phase $(\Delta \neq 0)$ increase dramatically, especially in the limit of $E_{f}<0$. For negative $E_{f}$, the stability region of excitonic phase rapidly reduces with increasing $\left|t_{d}^{\prime}\right|$, and at $t_{d}^{\prime} \sim-0.4$, it fully disappears. This is obviously a consequence of the absence of a mixed valence phase for $n_{f}>0.5$ and $t_{d}^{\prime} \leqslant-0.4$ as it is demonstrated in Fig 1 , where the
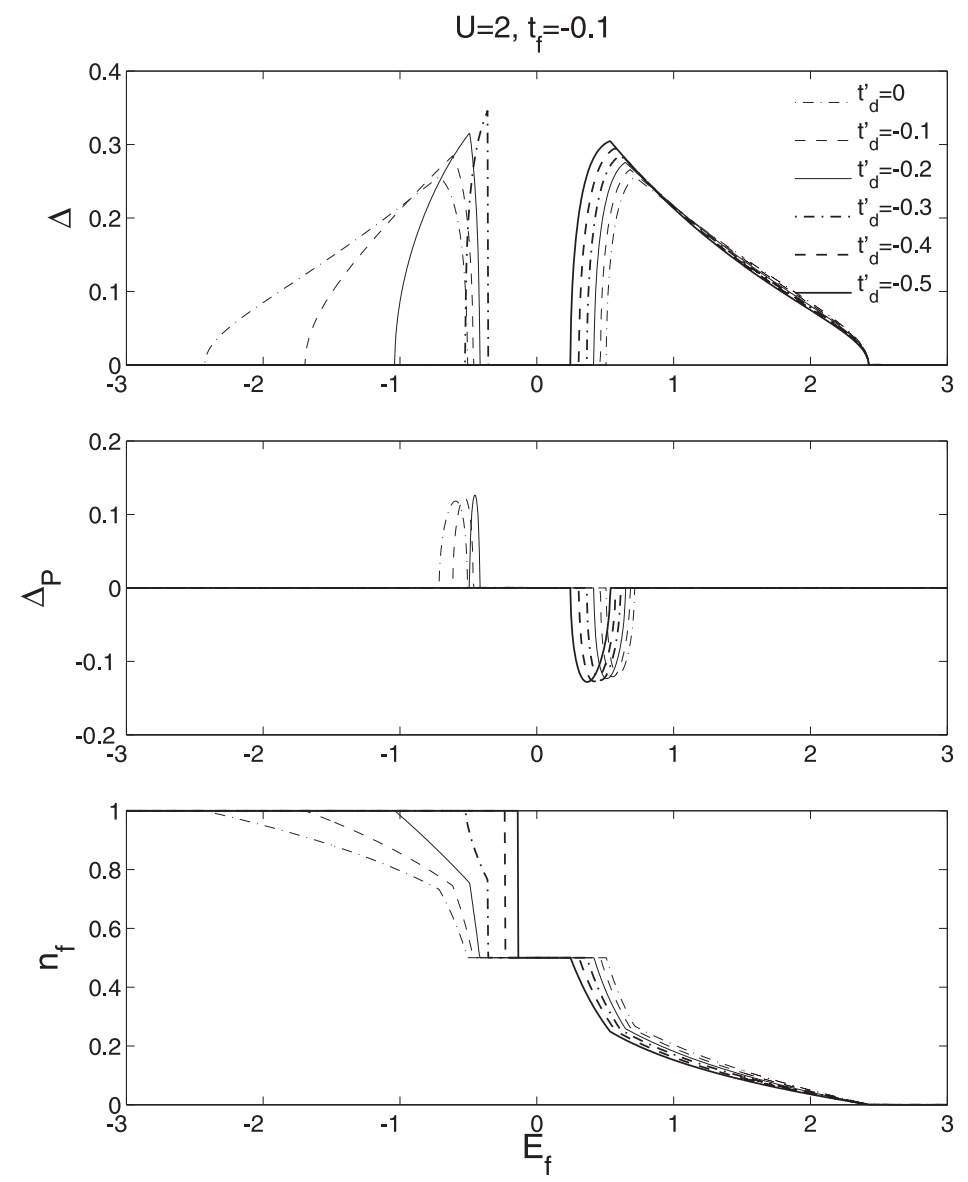

Figure 1. Dependence of the HF parameters $\Delta, \Delta_{P}$ and $n_{f}$ on the $f$-level energy $E_{f}$ calculated for different values of $t_{d}^{\prime}\left(t_{d}^{\prime}=0,-0.1,-0.2,-0.3,-0.4,-0.5\right)$ and $U=2, t_{f}=-0.1$. 
average $f$-electron occupancy $n_{f}$ is plotted as a function of the $f$-level energy $E_{f}$. In this case, the valence transitions change discontinuously from $n_{f}=1$ to $n_{f}=0.5$ and the system exhibits a direct transition from the BI phase to the CDW phase. At the same time, this result stresses the fact what a crucial role is played by the term of correlated hopping in the mechanism of valence transitions, and that already very small values of the correlated hopping parameter are capable of changing the type of valence transitions from continuous to discontinuous. For this reason, the term of correlated hopping should be surely taken into account in the correct description of valence transitions in rare-earth compounds.

In accordance with the case $t_{d}^{\prime}=0$, we have found two different excitonic phases for $t_{d}^{\prime}<0$. The first one is homogeneous $\left(\Delta>0, \Delta_{P}=0\right)$ and the second one is inhomogeneous $\left(\Delta>0, \Delta_{P} \neq 0\right)$. Analysing the numerical data obtained for $\delta_{d}, \delta_{f}$ and $\Delta_{P}$ (see figure 2), one can see that the appearance of an inhomogeneous phase is tightly connected with the formation of the CDW ordering in $f$ and $d$ electron subsystems since in the corresponding regions where $\Delta_{P} \neq 0$, the order parameter $\delta_{d}\left(\delta_{f}\right)$ changes continuously from 0 to its maximal (minimal) value. With increasing $\left|t_{d}^{\prime}\right|$, the stability region of the inhomogeneous phase strongly reduces and at $t_{d}^{\prime} \sim-0.3$ this phase practically disappears.
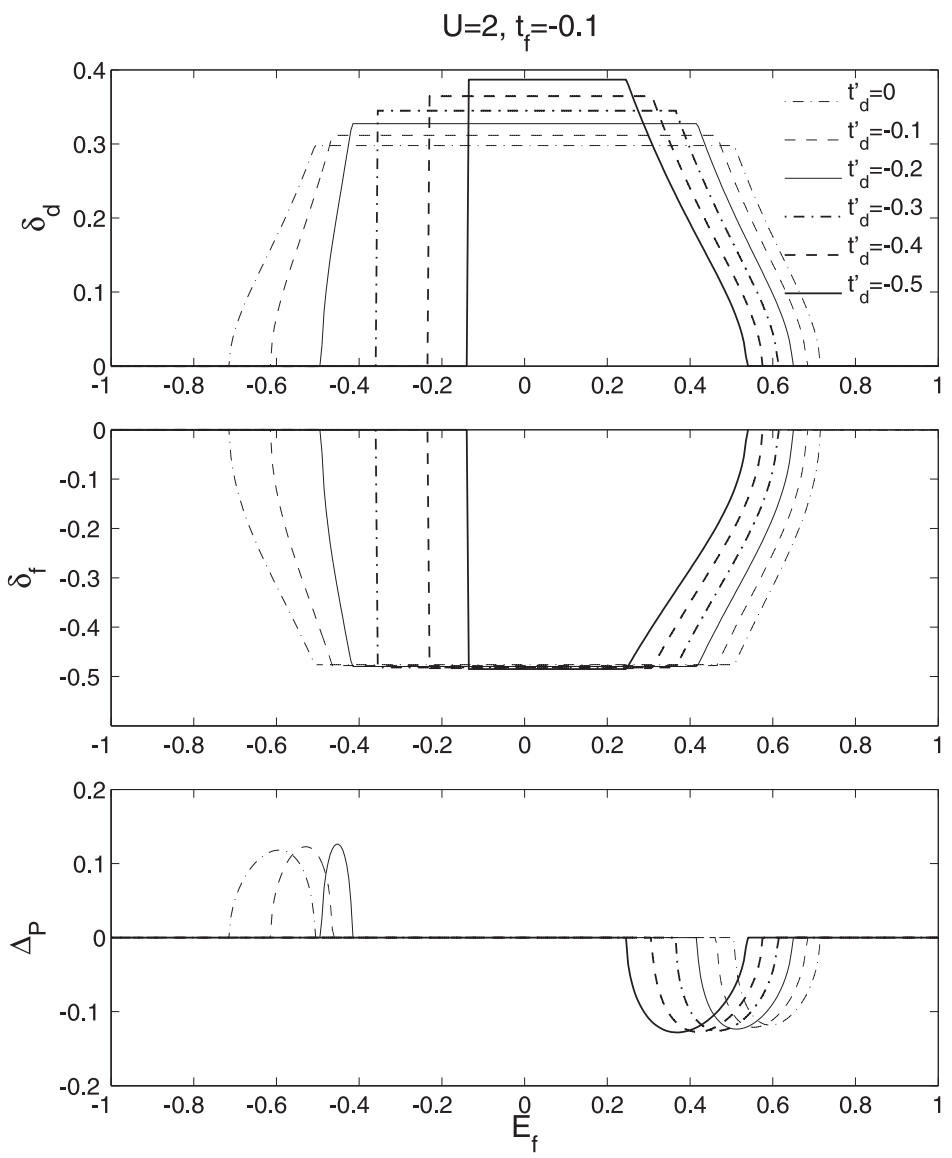

Figure 2. Dependence of the HF parameters $\delta_{d}, \delta_{f}$ and $\Delta_{P}$ on the $f$-level energy $E_{f}$ calculated for different values of $t_{d}^{\prime}\left(t_{d}^{\prime}=0,-0.1,-0.2,-0.3,-0.4,-0.5\right)$ and $U=2, t_{f}=-0.1$.

Going with $t_{d}^{\prime}$ to smaller values we have observed a completely different behavior of the model in the limit of $E_{f}<0$ (see figure 3). For all the examined values of $t_{d}^{\prime}$, we have found that $\Delta=0$ and $\Delta_{P}>0$, which means that the ground state of the model is antiferroelectric in this limit. Very interesting is also the behavior of $n_{f}$ as a function of $E_{f}$. As is shown in figure 4 the mixed-valent phase is again stabilized in the region $n_{f}>1 / 2$ and the magnitude of the discontinuous valence transition from $n_{f}>0.5$ to $n_{f}=1 / 2$ gradually decreases with decreasing $t_{d}^{\prime}$ and a similar behaviour also exhibits the width of the $n_{f}=1 / 2$ phase. At a first glance, this result seems to be in contradiction with the rules favour the formation of the electronically driven ferroelectric state postulated by Batista et al. [9]. The system is in a mixed-valence 

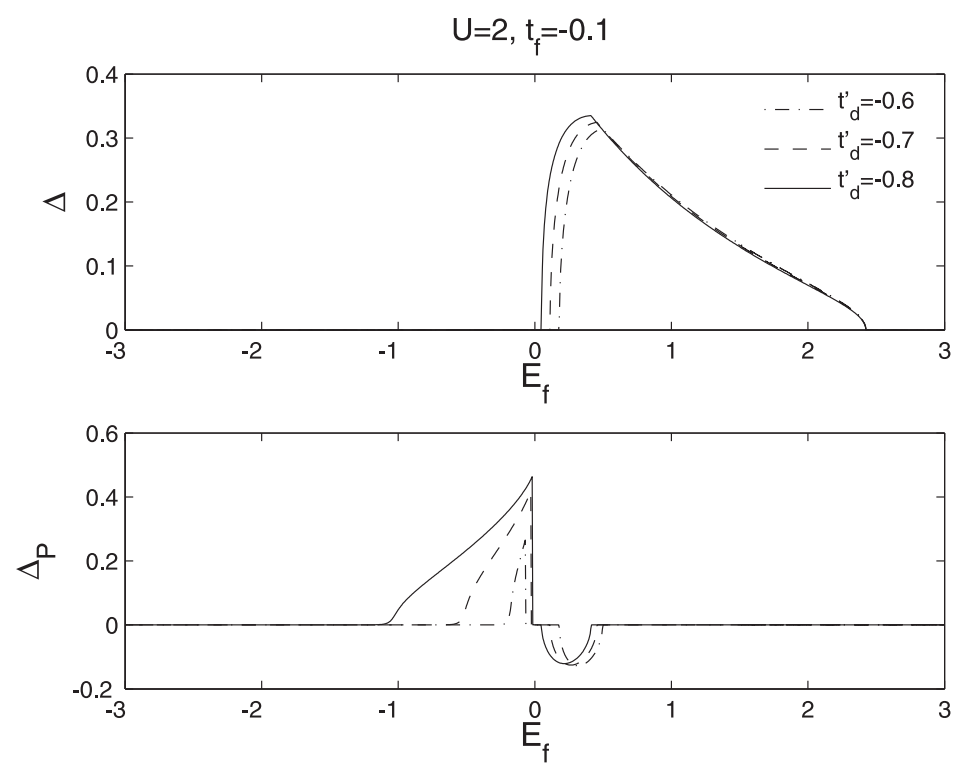

Figure 3. Dependence of the HF parameters $\Delta$ and $\Delta_{P}$ on the $f$-level energy $E_{f}$ calculated for different values of $t_{d}^{\prime}\left(t_{d}^{\prime}=-0.6,-0.7,-0.8\right)$ and $U=2, t_{f}=-0.1$.

regime, the local Coulomb interaction between different orbitals is present, but the ferroelectric state is absent. A more detailed analysis (see figure 4) of the behaviour of the renormalized $d$-electron hopping parameter $t_{d}^{*}$ shows, however, that there in no contradiction with conditions postulated by Batista et al., since for $t_{d}^{\prime}<-0.5$ and $E_{f}<0$, the $d$ band changes its parity. In this region, the $d$ and $f$ bands are of the same parity $\left(t_{d}^{*}<0, t_{f}<0\right)$, which, in accordance with the above mentioned rules, does not support the ferroelectric ground state.

The situation in the opposite limit $t_{d}^{\prime}>0$ is depicted in figure5, Again one can recognize two different regimes in the behaviour of $n_{f}, \Delta$ and $\Delta_{p}$. For positive $E_{f}$, only weak effects of correlated hopping on $n_{f}, \Delta$ and $\Delta_{p}$ are observed, while in the opposite limit, these effects are quite dramatic and the stability
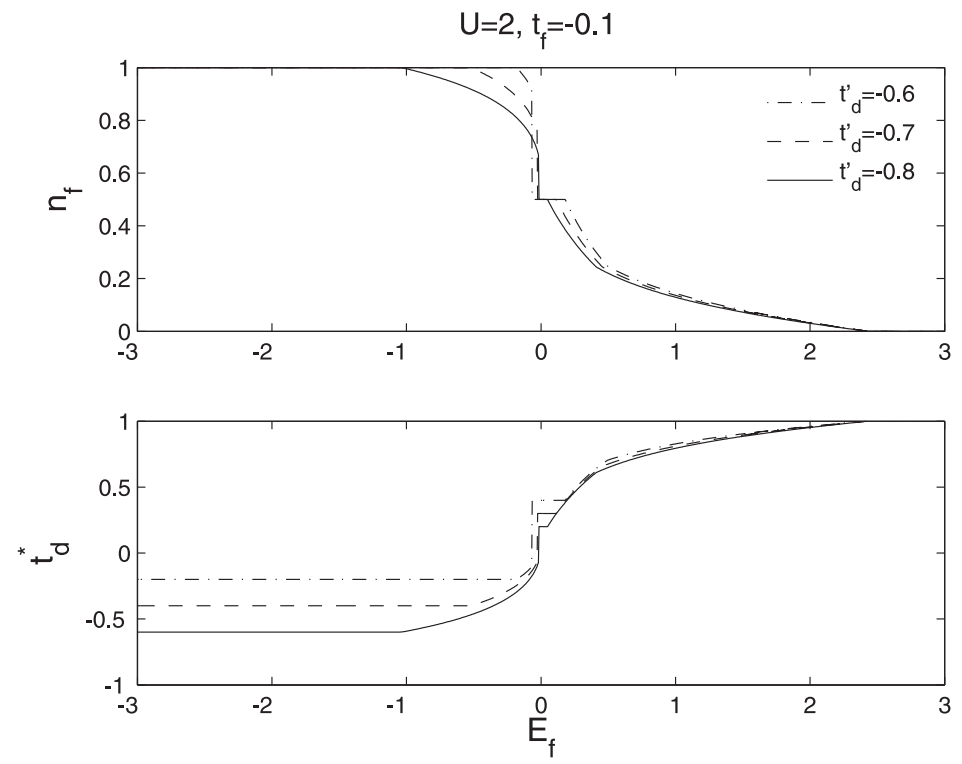

Figure 4. Dependence of the HF parameter $n_{f}$ and the renormalized $d$-electron hopping parameter $t_{d}^{*}$ on the $f$-level energy $E_{f}$ calculated for different values of $t_{d}^{\prime}\left(t_{d}^{\prime}=-0.6,-0.7,-0.8\right)$ and $U=2, t_{f}=-0.1$. 

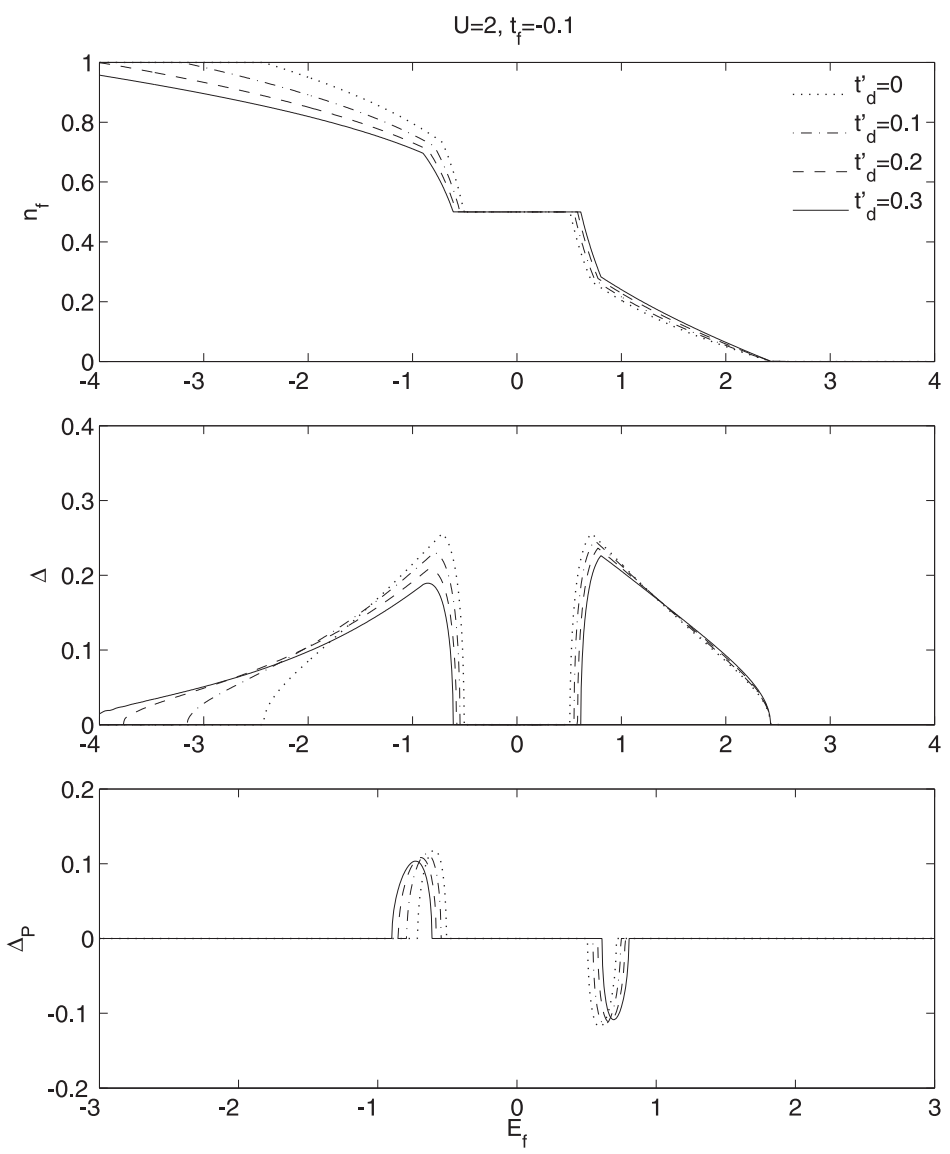

Figure 5. Dependence of the HF parameters $n_{f}, \Delta$ and $\Delta_{P}$ on the $f$-level energy $E_{f}$ calculated for different values of $t_{d}^{\prime}\left(t_{d}^{\prime}=0,0.1,0.2,0.3\right)$ and $U=2, t_{f}=-0.1$.

regions of the mixed valence as well as excitonic phase are considerably enhanced by increasing $t_{d}^{\prime}$. This result independently confirms that the term of correlated hopping plays a very important role in the mechanism of stabilizing the excitonic phase and, thus, it should be taken into account for the correct description of this phenomenon. Finally, it should be noted that we have also found qualitatively the same behaviour of the model for smaller values of $t_{f}$ and larger values of $U$.

Thus, we can conclude that the effects of correlated hopping term on the stability of the excitonic state in the extended FKM (within the HF approximation with the CDW instability) are very strong and they lead to: (i) suppression of the excitonic phase for $t_{d, \text { crit. }}^{\prime} \leqslant t_{d}^{\prime}<0, E_{f}<0$, (ii) stabilization of the antiferroelectric phase for $t_{d}^{\prime}<t_{d \text { crit. }}^{\prime}, E_{f}<0$ and (iii) stabilization of excitonic phase for $t_{d}^{\prime}>0, E_{f}<$ 0 . Similarly, the strong effects of correlated hopping are also observed on the mechanism of valence transitions, where already very small values of the correlated hopping parameter are capable of changing the type of valence transitions from continuous to discontinuous.

\section{Acknowledgements}

This work was supported by the Slovak Grant Agency VEGA under Grant No. 2/0077/13, Slovak Research and Development Agency (APVV) under Grant APVV-0097-12 and EU Grants No. ITMS26210120002 and ITMS26110230097. 


\title{
References
}

1. Falicov L.M., Kimball J.C., Phys. Rev. Lett., 1969, 22, 997; doi 10.1103/PhysRevLett.22.997

2. Portengen T., Östreich T., Sham L.J., Phys. Rev. Lett., 1996, 76, 3384; doi 10.1103/PhysRevLett.76.3384

3. Portengen T., Östreich T., Sham L.J., Phys. Rev. B, 1996, 54, 17452; doi 10.1103/PhysRevB.54.17452

4. Czycholl G., Phys. Rev. B, 1999, 59, 2642; doi 10.1103/PhysRevB.59.2642

5. Farkašovský P., Phys. Rev. B, 1999, 59, 9707; doi 10.1103/PhysRevB.59.9707.

6. Farkašovský P., Phys. Rev. B, 2002, 65, 81102; doi 10.1103/PhysRevB.65.081102.

7. Zlatić V., Freericks J.K., Lemanski R., Czycholl G., Philos. Mag. B, 2001, 81, 1443; doi 10.1080/13642810110066470

8. Batista C.D., Phys. Rev. Lett., 2002, 89, 166403; doi 10.1103/PhysRevLett.89.166403

9. Batista C.D., Gubernatis J.E., Bonča J., Lin H.Q., Phys. Rev. Lett., 2004, 92, 187601; doi 10.1103/PhysRevLett.92.187601

10. Farkašovský P., Phys. Rev. B, 2008, 77, 155130; doi 10.1103/PhysRevB.77.155130

11. Schneider C., Czycholl G., Eur. Phys. J. B, 2008, 64, 43; doi 10.1140/epjb/e2008-00273-y

12. Zenker B., Ihle D., Bronold F.X., Fehske H., Phys. Rev. B, 2010, 81, 115122; doi 10.1103/PhysRevB.81.115122

13. Phan V.N., Becker K.W., Fehske H., Phys. Rev. B, 2010, 81, 205117; doi 10.1103/PhysRevB.81.205117

14. Seki K., Eder R., Ohta Y., Phys. Rev. B, 2011, 84, 245106; doi 10.1103/PhysRevB.84.245106

15. Zenker B., Ihle D., Bronold F.X., Fehske H., Phys. Rev. B, 2012, 85, 121102R; doi 10.1103/PhysRevB.85.121102

16. Kaneko T., Seki K., Ohta Y., Phys. Rev. B, 2012, 85, 165135; doi 10.1103/PhysRevB.85.165135.

17. Kaneko T., Ejima S., Fehske H., Ohta Y., Phys. Rev. B, 2013, 88, 035312; doi 10.1103/PhysRevB.88.035312

18. Apinyan V., Kopec T.K., J. Low Temp. Phys., 2014, 176, 27; doi 10.1007/s10909-014-1165-X

19. Ejima S., Kaneko T., Ohta Y., Fehske H., Phys. Rev. Lett., 2014, 112, 026401; doi 10.1103/PhysRevLett.112.026401

20. Hubbard J., Proc. R. Soc. London A, 1963, 276, 238; doi 10.1098/rspa.1963.0204

21. Hirsch J.E., Physica C, 1989, 158, 236; doi 10.1016/0921-4534(89)90225-6

22. Leder H.J., Solid State Commum., 1978, 27, 579; doi 10.1016/0038-1098(78)90399-X

23. Brydon P.M.R., Zhu J.X., Gulacsi M., Bishop A.R., Phys. Rev. B, 2005, 72, 125122; doi 10.1103/PhysRevB.72.125122

\section{Вплив корельованого переносу на електронну сегнетоелектрику в розширеній моделі Фалікова-Кімбала у двох вимірах}

\author{
П. Фаркашовскі, Ю. Юречкова \\ Інститут експериментальної фізики, Словацька академія наук, 04001 Кошиці, Словаччина
}

Ми використовуємо наближення Гартрі-Фока (ГФ) із нестійкістю щодо хвиль зарядової густини до дослідження впливу корельованого переносу на стійкість електронної сегнетоелектрики в розширеній моделі Фалікова-Кімбала (ФК) у двох вимірах. Показано, що внесок корельованого переносу $є$ дуже сильний, особливо для від'ємних значень енергії $f$-рівня $E_{f}$, коли цей вплив приводить до: (1) стабілізації сегнетоелектричного основного стану зі спонтанною гібридизацією $P_{d f}=\left\langle d^{+} f\right\rangle$ для додатних значень параметра корельованого переносу $t_{d^{\prime}}^{\prime}$ (2) стабілізації антисегнетоелектричної фази при $t_{d}^{\prime}<t_{d \text {,crit. }}^{\prime}<0$ і (3) приглушення сегнетоелектричного основного стану для $t_{d, \text { crit. }}^{\prime} \leqslant t_{d}^{\prime}<0$. Крім того, обговорюються впливи корельованого переносу на переходи зі зміною валентності.

Ключові слова: електронна сегнетоелектрика, хвилі зарядової густини, переходи зі зміною валентності 